\title{
The developing human brain-modeled in a dish
}

Stem cells grown in three-dimensional cultures self-organize into tissues that resemble the developing human brain.

Among the most interesting findings in stem cell biology of recent years has been the realization that when stem cells are cultured in optimal conditions, they show an extraordinary capacity to self-organize into structures that resemble physiological tissues. Researchers in the field have coined the term 'organoid' to name these 'tissues in the dish' that contain different cell types and reproduce several aspects of the tissue's architecture. Using pluripotent stem cells that were practically left alone in the dish, researchers have so far made organoids similar to the intestines, liver, eyes and even parts of brains. Now recent work from Jürgen Knoblich's lab at the Institute of Molecular Biotechnology of the Austrian Academy of Science in Vienna adds to the list organoids that mimic the developing human brain.

In the study, led by postdoc Madeline Lancaster, the researchers developed a protocol to generate what they call "cerebral organoids" that was inspired by prior work in the field and that focused on improving growth conditions and providing the environment necessary for the cells' intrinsic cues to influence development. They took a human embryonic cell line and a human induced pluripotent cell line and obtained embryoid bodies-floating aggregates of cells-that were differentiated into neural tissue using specific media. Then they transferred the neural aggregates into threedimensional gel matrix droplets. Once the aggregates reached a certain size, they were placed in a bioreactor, where they were spun to enhance the flow of nutrients in the medium. Within a few weeks, this approach led to organoids that contained cell types and anatomical structures similar to those in the brain of a 9-week-old human fetus.

A key part of the process was that the team did not add any neuronal differentiation factors to the three-dimensional cultures until the very end. Although Lancaster did try adding differentiation factors earlier in the protocol, in those cases the organoids that she obtained were actually smaller. The team followed the division of neural precursor cells present in the organoids over time and found that these cells generated neurons in the stereotypical way that is characteristic of the mammalian cortex.

Both Knoblich and Lancaster see themselves as neurodevelopmental biologists, not bioengineers. Their goal when delving into this project was to obtain tools that would help answer specific scientific questions. Although their lab has traditionally studied genes important for cell division and neuronal differentiation in the fruit fly, they have, over the years, sought to extend those studies to higher organisms such as mice and, eventually, humans. But in order to move to humans, Knoblich and his team felt that they needed to develop a model that would allow them to experimentally manipulate the system and study it in detail.

It has been shown that developing human brains contain a unique type of neural stem cell precursor, and it is thought that these precursors divide using different mechanisms than the ones that have been described in flies or mice. Because the team was able to find these unique human precursors in the organoids, they can now figure out what makes them so special.

Despite the exciting advance that this model represents, it is still far from reproducing the brain as we know it-even a fetal one. Within the organoids, areas resembling different brain regions are distributed at random and do not replicate the spatial organization seen in the brain. The organoids also stop growing after a certain amount of time, and their interiors are filled with dead cells owing to the lack of a necessary circulatory system to deliver nutrients and oxygen throughout the tissue. The model is also not appropriate for studying brain circuits and their function, at least for now.

Yet the technology lends itself well to the study of many interesting cell biological

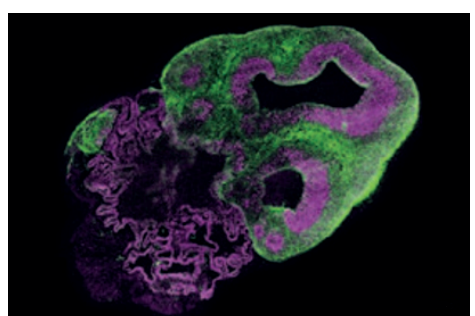

A cerebral organoid. Image courtesy of M. Lancaster and J. Knoblich.

processes, as it is amenable for experimental manipulation using molecular tools. In rodents, this type of culture platform could represent a bridge between classical neural culture experiments and those done in the brain in vivo. The organoids show great potential for disease modeling studies as well, particularly for studying diseases with a developmental component, including autism or schizophrenia. To demonstrate this, the researchers took fibroblasts from human patients with a neurodevelopmental disorder called microcephaly, which is characterized by a smaller-than-normal brain size, and generated organoids from these cells that were smaller than those obtained from normal cells and had reduced numbers of progenitors.

Knoblich looks back at the whole process thinking that, for a Drosophila lab, this type of project was courageous, and that without Lancaster's initiative it would have probably not gotten this far. "I think there are very few young scientists who can develop something like this, and I feel very lucky to have one of them in the lab." He also acknowledges that the work couldn't have happened without a funding source that was "high risk" and not dependent on getting positive results. "A project like this," he says, "requires funding that allows for failure."

\section{Erika Pastrana}

\section{RESEARCH PAPERS}

Lancaster, M.A. et al. Cerebral organoids model human brain development and microcephaly. Nature doi:10.1038/nature12517 (28 August 2013). 